\title{
Correction to: Goal-oriented error estimation and adaptivity in MsFEM computations
}

\author{
Ludovic Chamoin ${ }^{1,2}$. Frédéric Legoll ${ }^{3,4}$
}

Published online: 4 June 2021

(c) Springer-Verlag GmbH Germany, part of Springer Nature 2021

\section{Correction to: \\ Computational Mechanics (2021) 67:1201-1228 https://doi.org/10.1007/s00466-021-01990-x}

In the original publication of the article Fig. 29 was published incorrectly. The two images that are shown in Fig. 29 do not correspond to the caption given for this figure, nor to the model problem which we consider. They come from [1] and were actually used as a reference to check the code; they were accidentally inserted in the manuscript instead of the images we generated for our own model problem. The correct images, associated with the problem (permeability, loading, boundary conditions) addressed in Section 6.3, are given below. This mistake does not impact the numerical results displayed in Figures 30 and 31, nor the discussion carried out in Section 6.3.

The original article can be found online at https://doi.org/10.1007/ s00466-021-01990-x.

Ludovic Chamoin

ludovic.chamoin@ens-paris-saclay.fr

Frédéric Legoll

frederic.legoll@enpc.fr

1 Université Paris-Saclay, ENS Paris-Saclay, CNRS, LMT, 4 avenue des Sciences, 91190 Gif-sur-Yvette, France

2 Institut Universitaire de France (IUF), 1 rue Descartes, 75231 Paris Cedex 5, France

3 Laboratoire Navier (ENPC, Paris-Est University), 6-8 avenue Blaise Pascal, 77455 Marne-La-Vallée Cedex 2, France

4 Inria Paris, MATHERIALS project-team, 2 rue Simone Iff, CS 42112, 75589 Paris Cedex 12, France 

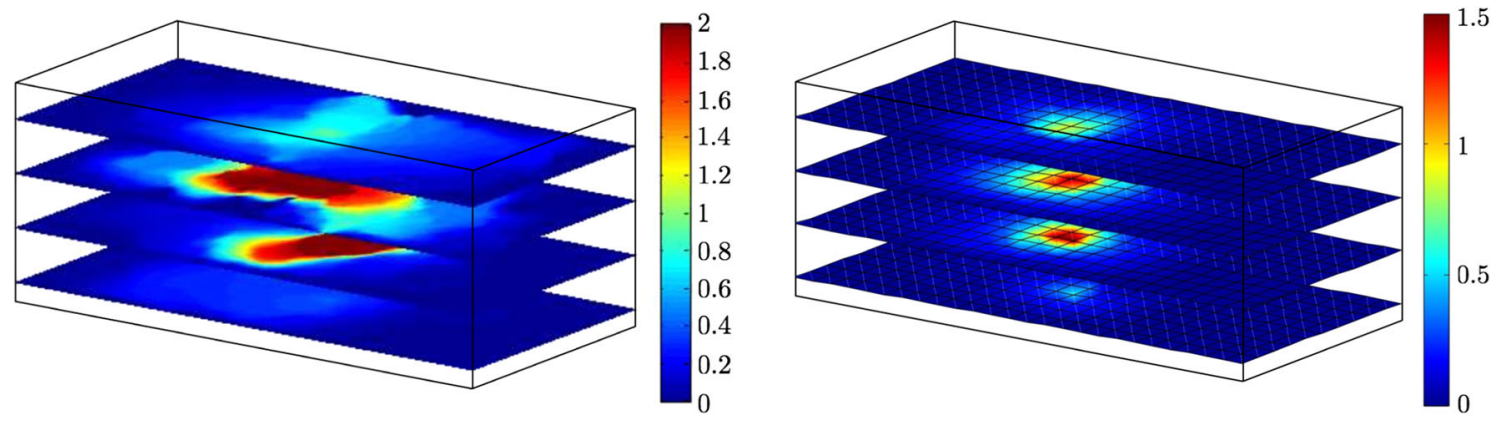

Fig. 29 Waterflood problem: exact solution $u^{\varepsilon}$ (left) and initial MsFEM solution $u_{H}^{\varepsilon}$ (right)

\section{Reference}

1. Christie MA, Blunt MJ (2001) Tenth SPE comparative solution project: a comparison of upscaling techniques. SPE Reservoir Evaluation and Engineering

Publisher's Note Springer Nature remains neutral with regard to jurisdictional claims in published maps and institutional affiliations. 